\title{
Selective expression of the scaffold protein JSAP1 in spermatogonia and spermatocytes
}

\author{
Munkhuu Bayarsaikhan ${ }^{1}$, Akiko Shiratsuchi ${ }^{2}$, Davaakhuu Gantulga ${ }^{1}$, Yoshinobu Nakanishi $^{2}$ \\ and Katsuji Yoshioka ${ }^{1}$ \\ ${ }^{1}$ Division of Cell Cycle Regulation, Cancer Research Institute, Kanazawa University, 13-1 Takara-machi, \\ Kanazawa, Ishikawa 920-0934, Japan and ${ }^{2}$ Graduate School of Medical Science, Kanazawa University, Shizenken, \\ Kakuma-machi, Kanazawa, Ishikawa 920-1192, Japan \\ Correspondence should be addressed to K Yoshioka; Email: katsuji@kenroku.kanazawa-u.ac.jp
}

M Bayarsaikhan and A Shiratsuchi contributed equally to this study

\begin{abstract}
Scaffold proteins of mitogen-activated protein kinase (MAPK) intracellular signal transduction pathways mediate the efficient and specific activation of the relevant MAPK signaling modules. Previously, our group and others have identified $\mathrm{c}_{-}$Jun $\mathrm{NH}_{2}-$ terminal kinase (JNK)/stress-activated protein kinase-associated protein 1 (JSAP1, also known as JNK-interacting protein 3) as a scaffold protein for JNK MAPK pathways. Although JSAP1 is expressed in the testis in adults, its expression during development has not been investigated. In addition, it is unknown which types of cells in the testis express the scaffold protein. Here, we examined the expression of JSAP1 in the testis of mice aged 14 days, 20 days, 6 weeks, and 12 weeks by immunohistochemistry and Western blotting. The specificity of the anti-JSAP1 antibody was evaluated from its reactivity to exogenously expressed JSAP1 and a structurally related protein, and by antigen-absorption experiments. The immunohistochemical analyses with the specific antibody showed that the JSAP1 protein was selectively expressed in the spermatogonia and spermatocytes, but not in other cell types, including spermatids and somatic cells, during development. However, not all spermatogonia and spermatocytes were immunopositive either, especially in the 12-week-old mouse testis. Furthermore, we found by Western blotting that the expression levels of JSAP1 protein vary during development; there is high expression until 6 weeks after birth, which approximately corresponds to the end of the first wave of spermatogenesis. Collectively, these results suggest that JSAP1 function may be important in spermatogenic cells during early postnatal development.

Reproduction (2006) 131 711-719
\end{abstract}

\section{Introduction}

The mature male gamete is produced through spermatogenesis, a complex process. The differentiation of spermatogenic cells into spermatozoa occurs within seminiferous tubules, where both spermatogenic and somatic cells cooperatively control the progressive steps through the mitotic, meiotic, and post-meiotic phases of development. Extrinsic signals, including endocrine and paracrine molecules, modulate spermatogenesis by regulating gene expression in the spermatogenic cells (Griswold 1998, Eddy 2002). Extensive studies have been carried out to understand spermatogenesis at the molecular level. In addition, comprehensive and systematic studies with highthroughput analyses have begun, and have resulted in the identification of novel genes that are potentially involved in spermatogenesis. Indeed, Shima et al. (2004) used microarrays to analyze testes from mice aged $0-56$ days at 11 time points, and they published the mRNA expression profiles of the developing testis. However, much remains to be learned about the molecular mechanisms that regulate spermatogenesis.

Mitogen-activated protein kinase (MAPK) intracellular signal transduction pathways are protein kinase cascades and play key roles in many cellular processes, including proliferation, differentiation, apoptosis, and migration (Widmann et al. 1999, Chang \& Karin 2001, Kyriakis \& Avruch 2001). Upon stimulation, the MAPK cascade transmits its signal through a phosphorylation relay via MAPK kinase kinase (MAP3K), MAPK kinase (MAPKK), and MAPK. The activated MAPK then modulates the activity of its effectors, e.g. transcription factors, by phosphorylating them. Thus, MAPK signaling cascades functionally connect extracellular stimuli and the corresponding regulation of gene expression in the nucleus. In mammals, three groups of MAPK pathways, c-Jun $\mathrm{NH}_{2}$-terminal kinase (JNK), p38, and extracellular signal-regulated kinase (ERK) cascades, have been extensively studied. The scaffold proteins of the MAPK cascades organize the MAPK signaling components 
into functional MAPK modules, thereby enabling the efficient activation of specific MAPK cascades (Morrison \& Davis 2003, Yoshioka 2004). Our group and others have identified JNK/stress-activated protein kinase-associated protein 1 (JSAP1, also known as JNK-interacting protein 3 (JIP3)) as a scaffold protein for JNK MAPK cascades (Ito et al. 1999, 2000, Kelkar et al. 2000). JNK-associated leucine zipper protein (JLP, also known as JIP4), a structurally related member of the JSAP1 family, is likely to function as a scaffold for p38 MAPK cascades (Lee et al. 2002, Kelkar et al. 2005). Since JSAP1 is expressed in the adult testis (Ito et al. 1999, Kelkar et al. 2000), it is speculated that JNK MAPK signaling pathways are involved in the regulation of gene expression during spermatogenesis. However, the expression of JSAP1 in the testis during development has not been analyzed, and it is unknown which types of cells in the testis express the scaffold protein.

In the present study, we examined the expression of JSAP1 in the testis of mice of different ages and found that JSAP1 protein is selectively expressed in spermatogonia and spermatocytes. Furthermore, we observed that the expression levels of JSAP1 protein vary during development, and are quite high until 6 weeks after birth, a time that approximately corresponds to the end of the first wave of spermatogenesis. Our results suggest that JSAP1 may play important roles in spermatogenic cells during early postnatal development.

\section{Materials and Methods}

\section{Cell culture}

Human 293T cells were cultured in Dulbecco's modified Eagle's medium (DMEM, Sigma) supplemented with $10 \%$ fetal bovine serum in a $5 \% \mathrm{CO}_{2}$ atmosphere at $37^{\circ} \mathrm{C}$. 293T cells $\left(1 \times 10^{5}\right)$ in a $35-\mathrm{mm}$ dish were transfected with plasmid DNA using FuGENE 6 (Roche Diagnostics) according to the manufacturer's instructions. Thirty-six hours after the transfection, the cells were harvested and used for Northern and/or Western blotting analyses.

\section{Expression plasmids}

To generate a mammalian expression plasmid for Flagtagged JSAP1, pcDNA3-His-S-JSAP1 (Ito et al. 1999) was digested with EcoRI and Xhol, and the EcoRI-Xhol fragment containing the entire coding sequence of mouse JSAP1 was subcloned into EcoRI/Xhol-digested pcDNA3-Flag (Ito et al. 1999). The resultant plasmid was termed pcDNA3-FlagJSAP1. The coding region of the mouse JLP cDNA was amplified from BALB/c mouse brain CDNA by polymerase chain reaction (PCR). The product (containing an EcoRI site at the $5^{\prime}$ end and a stop codon followed by a Xhol site at the $3^{\prime}$ end of the sense strand) was digested with EcoRI and Xhol, and subcloned into EcoRI/Xhol-digested pcDNA3Flag to generate pcDNA3-Flag-JLP. The structure of the plasmid construct was verified by restriction enzyme map- ping and nucleotide sequencing. To generate an expression plasmid for the JSAP1 glutathione S-transferase (GST)fusion protein, the region encoding amino acid residues 115-504 of JSAP1 was amplified by PCR. The product (containing a BamHI site at the $5^{\prime}$ end and a stop codon followed by a Xhol site at the $3^{\prime}$ end of the sense strand) was digested with BamHI and Xhol and subcloned into Bam$\mathrm{HI} /$ Xhol-digested pGEX-4T-3 (Amersham Biosciences) to generate pGEX-JSAP1(115-504). Among four JSAP1 isoforms, termed JSAP1a-d, which are generated through alternative splicing (Ito et al. 2000), the JSAP1a isoform was used for constructing the mammalian and bacterial expression plasmids.

\section{Antibodies}

The GST-JSAP1 (residues 115-504) protein was expressed in Escherichia coli and purified with glutathione Sepharose (Amersham Biosciences) according to the manufacturer's instructions. The purified protein was injected into $\mathrm{Kbl}: J \mathrm{~W}$ rabbits and the serum was collected for testing. The antiserum was first absorbed on an immobilized GSTSepharose column, and the flow-through fraction was further purified with the aid of an antigen-affinity column, the GST-JSAP1(115-504)-Sepharose column. The anti-Flag M2 monoclonal antibody and the anti-actin polyclonal antibody were purchased from Sigma. The antibody to actin (cat. no. A5060) shows a broad reactivity with actin isoforms and across a range of organisms. The donkey anti-rabbit horseradish peroxidase-conjugated secondary antibody was obtained from Amersham Biosciences.

\section{Northern blotting}

Total RNAs were prepared using Sepasol-RNAI (Nakalai Tesque, Kyoto, Japan) according to the manufacturer's instructions. Northern blot analysis was performed as described previously (Sato et al. 2004). The 0.5-kilobase (kb) fragment of mouse JSAP1, corresponding to amino acid residues 191-350 was used as the probe. Mouse glyceraldehyde-3-phosphate dehydrogenase (GAPDH) cDNA was amplified by PCR with the primers 5'-TGAAGGTCGGTGTGAACGGATTTGGC- $3^{\prime}$ and $5^{\prime}$-CATGTAGGCCATGAGGTCCACCAC- $3^{\prime}$, and the resultant 1 -kb fragment was used as the probe.

\section{Western blotting}

Mouse tissues or cultured cells were lysed in the following buffer: $50 \mathrm{mM}$ Tris- $\mathrm{HCl}$ ( $\mathrm{pH} 7.4), 150 \mathrm{mM} \mathrm{NaCl}, 2 \mathrm{mM}$ EDTA, $10 \%$ glycerol, and $1 \%$ SDS, protease inhibitor cocktail (Sigma). The extracts were precleared by centrifugation $\left(12000 \times \mathbf{g}\right.$ at $4{ }^{\circ} \mathrm{C}$ for $\left.10 \mathrm{~min}\right)$, and the supernatants were used as protein samples. Western blot analysis was carried out as described previously (Sato et al. 2004). In cases that required the quantification of proteins on immunoblots, the propriety software, Science Lab 2001 Image Gauge (Fujifilm, Tokyo, Japan) was used. 


\section{Animals}

All the mice used in this study were male ddY mice. Twenty-day-old Donryu rats were used for the isolation of Sertoli cells and spermatogenic cells. All experiments involving animals were conducted with the approval of the Committee on Animal Experimentation of Kanazawa University.

\section{Preparation of testicular cells}

Cells were isolated from the seminiferous tubules of 20day-old Donryu rats and the primary cultures were grown for 3 days as described previously (Nagao 1989, Shiratsuchi et al. 1999). Spermatogenic cells were detached from the Sertoli cell monolayer by gentle pipetting and collected by centrifugation. The remaining Sertoli cells were detached from the culture containers by scraping and collected by centrifugation. Both cell types were obtained with a purity of about $90 \%$.

\section{Immunohistochemistry}

Testes and epididymis were dissected from ddY mice and fixed by soaking in Bouin's solution at $4{ }^{\circ} \mathrm{C}$ overnight. The fixed tissues were rinsed with phosphate-buffered saline (PBS), dehydrated, embedded with paraffin, and sectioned at $5 \mu \mathrm{m}$ thickness. The sections were placed on silanecoated glass slides, deparaffinized with xylene, dehydrated with ethanol, and washed with PBS. They were then microwaved $(500 \mathrm{~W})$ in $0.1 \mathrm{M}$ sodium citrate $(\mathrm{pH} 6.0)$ for 4 min for antigen retrieval, and then chilled in ice-cold PBS. The sections were successively incubated with $0.3 \%$ $(\mathrm{w} / \mathrm{v})$ hydrogen peroxide to inactivate the endogenous peroxidase, blocked with $10 \%(\mathrm{v} / \mathrm{v})$ fetal bovine serum, and incubated with the anti-JSAP-1 antibody. They were subsequently reacted with biotinylated anti-rabbit IgG antibody (Vector, Burlingame, CA, USA), followed by treatment with horseradish peroxidase-conjugated streptavidin (Zymed, San Francisco, CA, USA). The samples were finally supplemented with a coloring solution $(50 \mathrm{mM}$ Tris- $\mathrm{HCl}(\mathrm{pH} 7.6), 0.002 \%$ hydrogen peroxide, and $0.1 \mathrm{mg} / \mathrm{ml}$ of 3,3-diaminobenzidine tetrahydrochloride) for signal visualization, counter-stained with either Meyer's hematoxylin or methyl green, dehydrated, coverslipped with Entellan New (Merck, Darmstadt, Germany), and examined under a microscope. The experiments in Figs 3 and 4 were performed independently at least three times, and representative results are shown.

\section{Results}

\section{Probe specificity and expression of JSAP1 in adult testis}

We first evaluated the specificity of the probes used in this study, a 0.5-kb JSAP1 cDNA fragment and an anti-JSAP1 antibody, using cells transiently transfected with the expression plasmid for either Flag epitope-tagged JSAP1 or JLP. As shown in Fig. 1A (upper panel, lanes 1 and 2), the cDNA probe detected the Flag-JSAP1 transcript, but not the Flag-JLP transcript. The similar expression levels of these transcripts in the cells were indirectly confirmed by Western blotting with the anti-Flag antibody (Fig. 1A lower panel, lanes 1 and 2). Likewise, the anti-JSAP1 antibody reactivity was restricted to the Flag-JSAP1 protein, and it did not recognize the Flag-JLP protein, even though the Flag-tagged proteins were expressed at comparable levels (Fig. 1B, lanes 1 and 2). These results indicate that the cDNA and antibody probes specifically recognize the JSAP1 mRNA and protein respectively. We then analyzed testis samples from 12-week-old mice by Northern and Western blotting with the same set of probes. Since JSAP1 is expressed abundantly in mouse brain, we used brain samples as controls. As shown in Fig. $1 \mathrm{~A}$ and B (right panels), JSAP1 mRNA and protein were detected in the testis of the 12-week-old mouse, in which the length and the apparent molecular weight of the JSAP1 products are indistinguishable from those in the brain (Fig. 1A and B, lanes 3 and 4).

\section{Expression of JSAP1 in spermatogenic cells}

We next asked whether JSAP1 is expressed in spermatogenic cells. For this purpose, spermatogenic and Sertoli cells were prepared from seminiferous tubules of 20-dayold rats, and were analyzed by Northern and Western blot analyses. JSAP1 mRNA was detected in both the spermatogenic and Sertoli cells at comparable levels (Fig. 2A). However, the relative expression levels of the JSAP1 protein in these cell types were quite different; expression was high in the spermatogenic cells, and there was no (or very low) expression in the Sertoli cells (Fig. 2B).

\section{Immunohistochemical analysis of JSAP1 in adult testis}

We examined whether the anti-JSAP1 antibody used for Western blotting could also be used for immunohistochemical analysis as a specific probe (Fig. 3A). For this purpose, we performed antigen-absorption experiments with testis sections from 12-week-old mice that were fixed in Bouin's solution. Immunopositive signals observed within seminiferous tubules were significantly lower in the presence of the GST-JSAP1 protein antigen (compare top and middle panels). In contrast, the control GST protein had little or no effect on the immunostaining under the same assay conditions (bottom panel). These results strongly suggest that the antibody specifically detects the JSAP1 protein in immunohistochemical analysis, as well as in Western blots. The immunopositive signals on the testis sections were evident in the spermatogonia and spermatocytes, which were identified by their morphology, counterstaining with hematoxylin, and the 
A
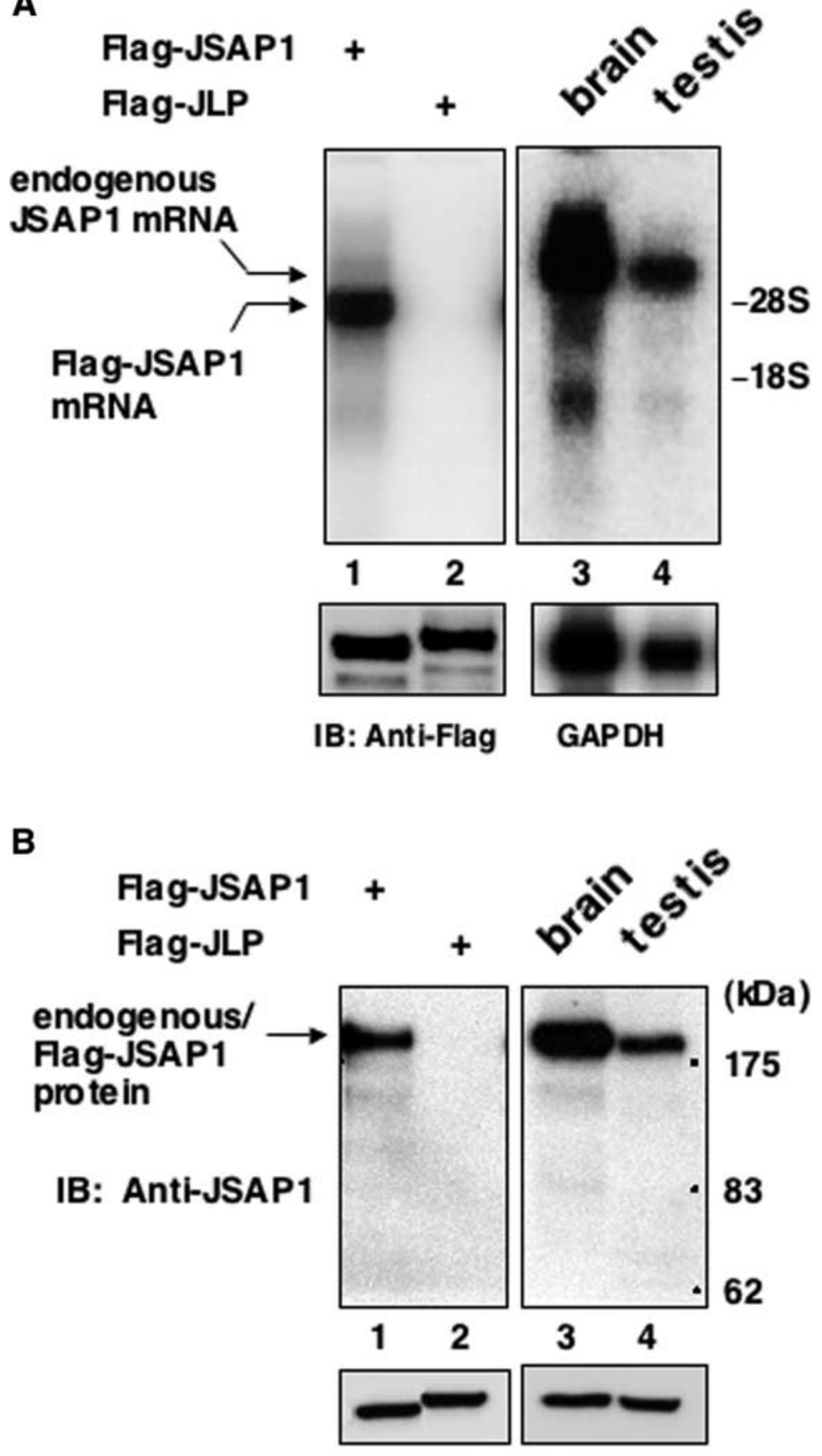

IB: Anti-Flag IB: Anti-Actin

Figure 1 Specificity of probes and expression of JSAP1 in mouse testis. (A) 293T cells were transiently transfected with $1 \mu \mathrm{g}$ pcDNA3-Flag-JSAP1 or pcDNA3-Flag-JLP as indicated. Total RNAs $(2.4 \mu \mathrm{g} /$ lane) were extracted from the cells and analyzed by Northern blotting with a $0.5-\mathrm{kb}$ ${ }^{32}$ P-labeled JSAP1 cDNA probe (upper panel, lanes 1 and 2). To confirm the expression of the exogenous JSAP1 and JLP in the cells, total cell lysates ( $4 \mu \mathrm{g} /$ lane) were subjected to immunoblotting (IB) with anti-Flag M2 antibody (lower panel, lanes 1 and 2). Total RNAs (5.4 $\mu \mathrm{g} /$ lane) prepared from the brain and testis of a 12-week-old mouse were analyzed by Northern blotting with the same probe (upper panel, lanes 3 and 4). The blot was reprobed with a GAPDH cDNA as a control (lower panel, lanes 3 and 4). (B) 293T cells were transiently transfected as in A, and the total cell lysates ( $20 \mu \mathrm{g} / \mathrm{lane}$ ) were subjected to immunoblotting with an anti-JSAP1 antibody (upper panel, lanes 1 and 2) or the anti-Flag M2 antibody (lower panel, lanes 1 and 2). Total cell lysates ( $20 \mu \mathrm{g} / \mathrm{lane})$ from brain and testis of a 12-week-old mouse were analyzed by Western blotting with the anti-JSAP1 antibody (upper panel, lanes 3 and 4). The blot was reprobed with an anti-actin antibody as a control (lower panel, lanes 3 and 4). The positions of the $28 \mathrm{~S}$ and $18 \mathrm{~S}$ ribosomal RNAs are given on the right in (A) and protein size markers are indicated on the right in (B). Experiments were performed independently three times with similar results.

positions of the cells in the seminiferous tubules (Fig. 3B). No positive signals were seen in the other cell types, including the spermatids and the somatic Leydig and Sertoli cells. The immunopositive spermatogenic cells were likely to be type A and B spermatogonia, and leptotene, zygotene, and pachytene spermatocytes, suggesting that the JSAP1 protein is expressed during the spermatogenic process from the primitive spermatogonium to the late prophase of meiosis I.
Immunohistochemical and Western blotting analyses of JSAP1 expression in the testis during development

To examine the expression of JSAP1 protein in the testis during development, we analyzed testis sections from mice aged 14 days, 20 days, 6 weeks, and 12 weeks by immunohistochemistry (Fig. 4). As with the testis sections from 12-week-old mice (Fig. 3B), the JSAP1 immunoreactivity was evident in spermatogonia and spermatocytes 

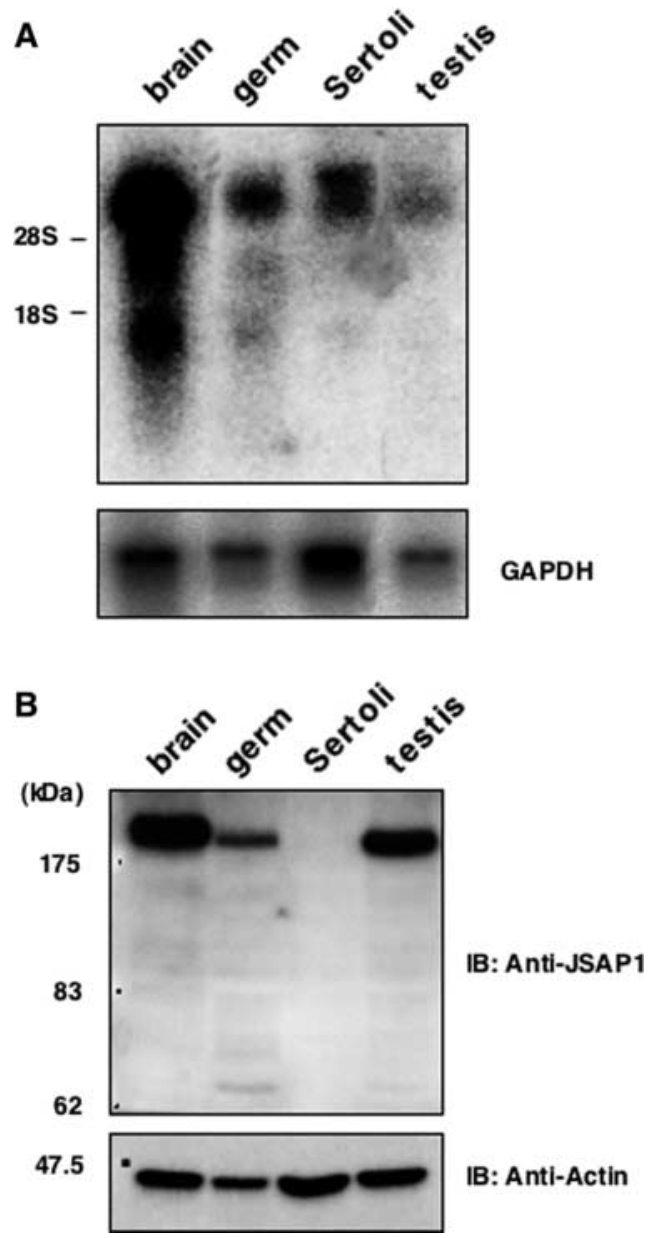

Figure 2 Expression of JSAP1 in spermatogenic cells. (A) Total RNAs $(5.4 \mu \mathrm{g} /$ lane) prepared from the brain and testis of a 20-day-old rat and from cultured rat spermatogenic (germ) and Sertoli cells were analyzed by Northern blotting with the ${ }^{32}$ P-labeled JSAP1 CDNA probe (upper panel). The positions of $28 \mathrm{~S}$ and $18 \mathrm{~S}$ ribosomal RNAs are indicated on the left. GAPDH mRNA was included as a control (lower panel). (B) Total cell lysates $(20 \mu \mathrm{g} / \mathrm{lane})$ from the brain and testis of a 20-day-old rat and from cultured rat spermatogenic (germ) and Sertoli cells were analyzed by immunoblotting (IB) with the antiJSAP1 antibody (upper panel). The positions of protein size markers are indicated on the left. The expression of actin is shown as a control (lower panel). The cultured rat cells were divided, and half were used for Northern blotting, and half for immunoblotting. Experiments were performed independently three times, and representative results are shown.

during development, and no positive signals were observed in other cell types. However, not all the spermatogonia and spermatocytes were immunopositive, especially in the sections from 12-week-old mice. In some sections, seminiferous tubules with no positive signals were observed, although the proportion of such tubules was low (data not shown). We also performed Western blotting analysis of testis samples from mice of the same ages (Fig. 5). The relative expression levels, normalized to the levels of actin and compared with the level at postnatal day 14 , were 1-, 2-, 0.5-, and 0.2-fold, in order of age. The expression level of JSAP1 was highest on postnatal day 20, and it remained relatively high until 6 weeks of age, which approximately corresponds to the end of the first wave of spermatogenesis. The lowest expression level was in the 12-week-old mouse.

\section{Discussion}

In the present study, we examined the expression of the scaffold protein JSAP1 in testes from mice of different ages by immunohistochemical and immunoblotting analyses. To our knowledge, JSAP1 is the first scaffold protein of the MAPK cascades whose expression has been analyzed in the testis during development.

The specificity of the probe is critical for expression analyses, especially if structurally related family members exist, as is true for JSAP1, which is structurally related to JLP (also called JIP4) (Lee et al. 2002, Kelkar et al. 2005). Furthermore, sperm-associated antigen 9 (SPAG9), a splice variant of JLP, is exclusively expressed in the testis and is present on the surface of mature spermatozoa (Jagadish et al. 2005). Therefore, our first step was to determine the specificity of our probes, a JSAP1 cDNA fragment and an anti-JSAP1 antibody. We tested their reactivity to exogenously expressed JSAP1 and JLP, and/or used them in antigen-absorption experiments. Our results showed that the probes were indeed specific for JSAP1 mRNA and protein in Northern blotting, Western blotting, and immunohistochemical analyses. Thus, it is quite reasonable to assume that the results obtained with the probes correctly reflect the expression of JSAP1.

We found by immunohistochemical analyses that JSAP1 protein is selectively expressed in spermatogonia and spermatocytes in mouse testes at different ages: 14 days, 20 days, 6 weeks, and 12 weeks. The expression patterns indicated that JSAP1 protein is likely to be expressed during the whole process of spermatogenesis, from the type A spermatogonium to the late prophase of the first meiosis. No positive signals were observed in other cell types, including spermatids and somatic cells, during development. In addition, JSAP1 protein was not detected in the ductus epididymidis (data not shown). JSAP1 protein, therefore, may play important roles in the early stages of spermatogenesis. However, even the spermatogonia and spermatocytes were not all immunopositive, especially in the sections from adult, 12-week-old mice. In addition, we showed by Western blotting that the expression levels of the JSAP1 protein in testis vary during development - of the ages tested it was highest in 20-dayold mice and remained relatively high until 6 weeks after birth. Taking into consideration that spermatogonia and spermatocytes appear, respectively, around postnatal days 11-14 and 21, and the time required for spermatozoa to differentiate from stem cells is $\sim 5$ weeks in mice, JSAP1 protein is likely to be expressed predominantly during the first wave of spermatogenesis. However, because there is 

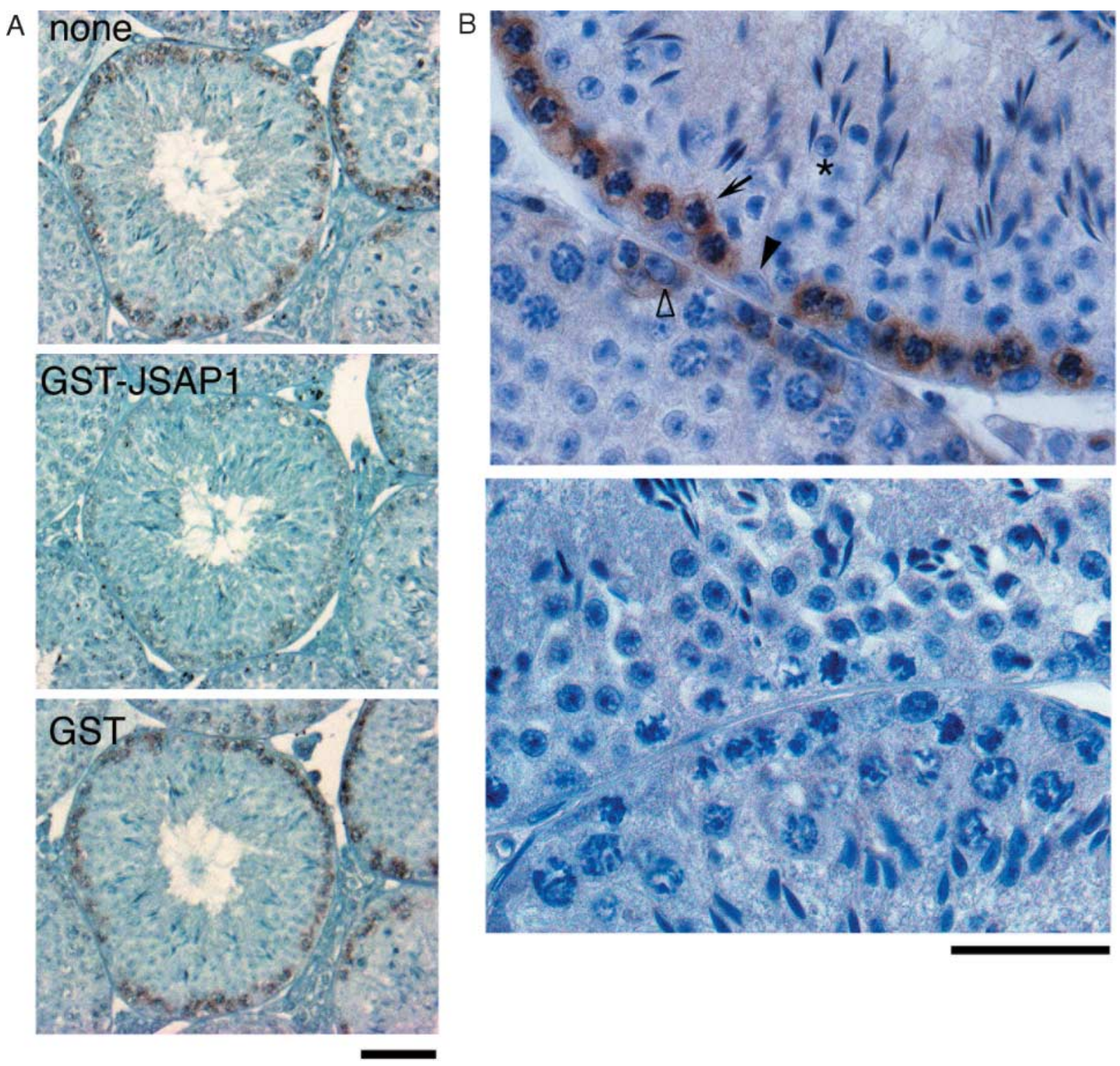

Figure 3 (A) Specificity of the anti-JSAP1 antibody in immunohistochemical analysis. Sections from the testis of a 12-week-old mouse testis were fixed in Bouin's solution and probed with the anti-JSAP1 antibody. The antibody was preincubated for 30 min without (top panel) or with $10 \mu \mathrm{g}$ of the GST-JSAP1 protein antigen (middle panel) or the control GST protein (bottom panel), and then used for the immunostaining experiments. All the sections were counterstained with methyl green. The immunopositive signals (brown) observed in peripheral cells within the seminiferous tubules were significantly reduced by absorption of the antibody with the GST-JSAP1 protein antigen, but not with the control GST protein. Scale bar represents $250 \mu \mathrm{m}$. (B) JSAP1 immunoreactivity in the testis of a 12-week-old mouse. Sections treated with Bouin's fixative were incubated with (upper panel) or without (lower panel) the anti-JSAP1 antibody, and the antigen-antibody complexes were detected with diaminobenzidine staining (brown). The section was counterstained with hematoxylin. The positive signals were seen in the spermatogonia (open arrowhead) and spermatocytes (arrow), but not in Sertoli cells (solid arrowhead) or spermatids (*). Scale bar represents $250 \mu \mathrm{m}$.

a massive expansion in the number of spermatogenic cells as the testis develops, and the relative expression of JSAP1 protein, which is expressed in spermatogonia and spermatocytes, should decrease accordingly, we cannot completely rule out the possibility that JSAP1 expression is stage-dependent and the expression levels remain constant in the JSAP1-expressing cells during spermatogenesis in mice, including the adult stage.
In Sertoli cells, there was no correlation between the expression of JSAP1 mRNA and protein (Fig. 2). In contrast to the JSAP1 mRNA, its translated product was not detectable by Western blotting. The lack of JSAP1 protein may indicate that JSAP1 expression is regulated post-translationally in the cells and JSAP1 protein is unstable due to its rapid degradation through the ubiquitin-proteasome system, as reported for rat pheochromocytoma PC12 $\mathrm{h}$ 

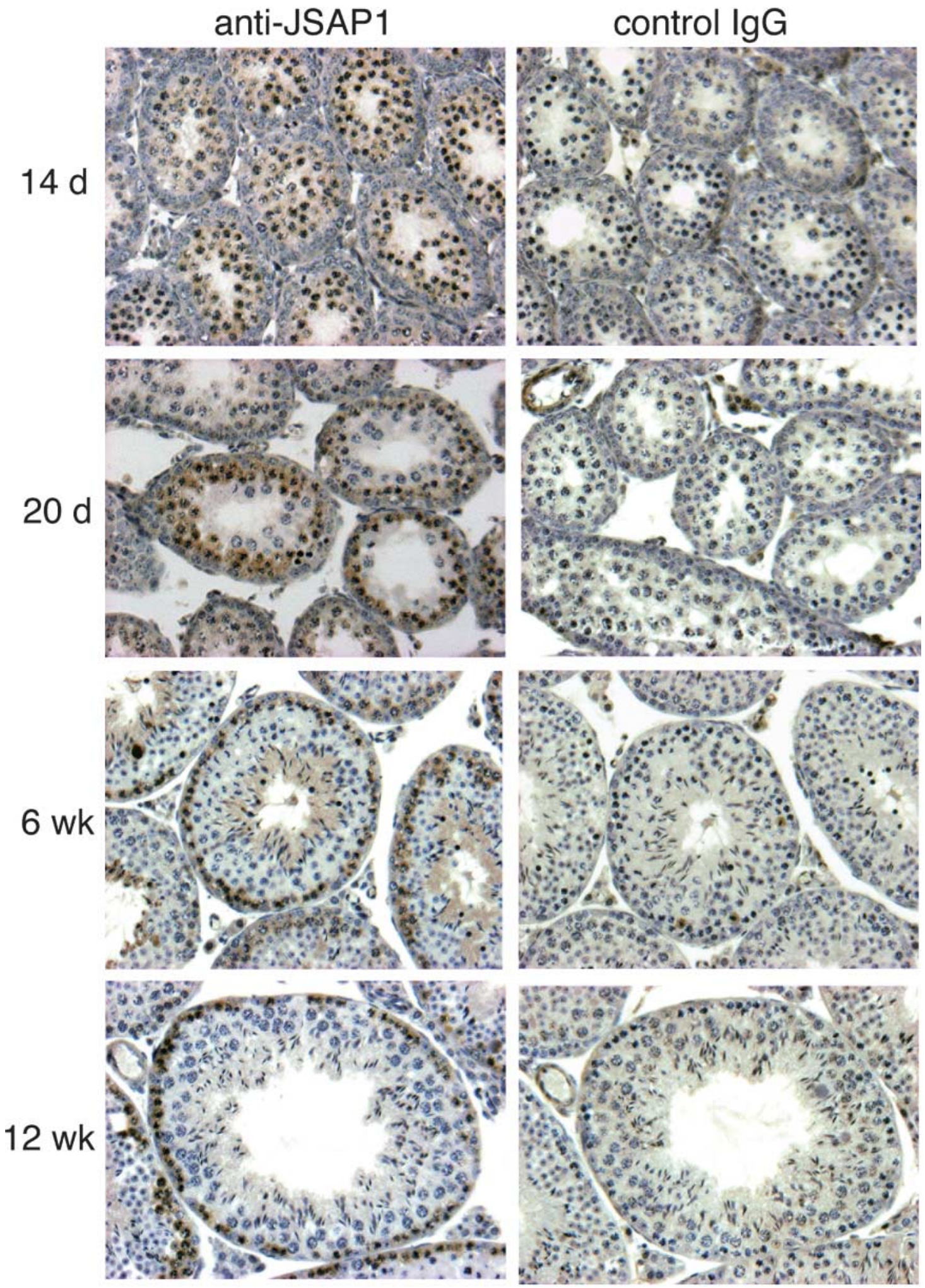

Figure 4 Immunohistochemical analysis of JSAP1 expression in the testis during development. Sections from the testis of mice aged 14 and 20 days (d) and 6 and 12 weeks (wk) were fixed in Bouin's solution and incubated with the anti-JSAP1 antibody (left panels) or control rabbit lgG (right panels). The antigen-antibody complexes were detected as in Fig. 3B. All the sections were counterstained with hematoxylin. Expression of JSAP1 was seen in sections from mice of all the ages tested. The only immunopositive cells were spermatogonia and spermatocytes. No positive signals were seen in the other cell types, including the spermatids and somatic cells. Scale bar represents $250 \mu \mathrm{m}$. 

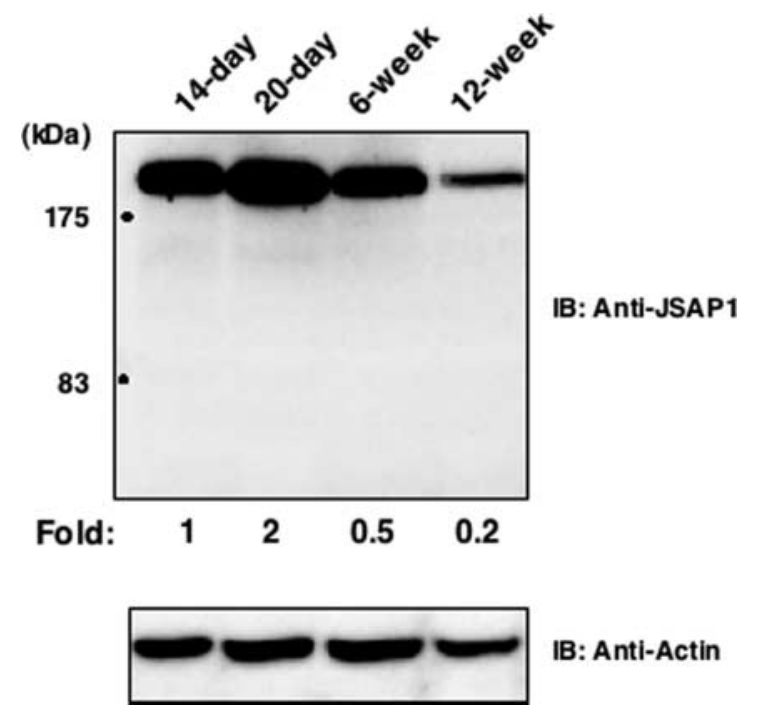

Figure 5 Western blot analysis of JSAP1 expression in the testis during development. Total cell lysates $(20 \mu \mathrm{g} / \mathrm{lane})$ from the testis of mice aged 14 days, 20 days, 6 weeks, and 12 weeks were subjected to immunoblotting (IB) with the anti-JSAP1 antibody (upper panel). The positions of protein size markers are indicated on the left. The expression of actin is included as a control (lower panel). The relative expression of JSAP1 at the different ages, normalized to the expression levels of actin, is given below the upper panel. Experiments were carried out independently three times with similar results.

cells (Sato et al. 2004). Since post-transcriptional regulation of gene expression is critical during spermatogenesis (Hecht 1986, Elliott 2003), it is also possible that the translation of JSAP1 mRNA is repressed in Sertoli cells. Further studies will be necessary to clarify this issue.

Type-A spermatogonia are self-renewing as well as capable of generating differentiating cells. We previously reported that in vitro neural differentiation through embryoid body (EB) formation is impaired in JSAP1-null cells, in which the number of neurites that differentiate from a given number of EBs is drastically decreased ( $\mathrm{Xu}$ et al. 2003). Taking this finding together with our current data, we speculate that JSAP1 might contribute to the regulation of self-renewal and/or differentiation of both spermatogonia and neural stem cells by modulating JNK signaling pathways. Hence, it would be interesting to investigate whether JSAP1/JNK pathways are involved in the regulation of the gene expression of Plzf, a transcriptional repressor essential for spermatogonial self-renewal (Buaas et al. 2004, Costoya et al. 2004). We also speculate that JSAP1/JNK pathways might regulate critical events, such as synaptonemal complex formation and DNA repair, during spermatogenesis.

The gene knockout approach is useful for clarifying the physiological role of molecules in vivo. However, this strategy cannot be applied straightforwardly to JSAP1, because JSAP1-deficient mice die shortly after birth, most likely due to a failure to breathe (Kelkar et al. 2003, Ha et al. 2005). To overcome this problem and to uncover the physiological importance of JSAP1 during spermatogenesis, a spermatogenic cell-specific JSAP1 knockout, especially a knockout that is effective in the early stages of postnatal development, will be required. We are currently attempting this knockout strategy.

\section{Acknowledgements}

We thank Dr H Tanahashi (Kanazawa University) for helpful discussion. This research was supported, in part, by a Grantin-Aid for Scientific Research from the Ministry of Education, Culture, Sports, Science and Technology of Japan. The authors declare that there is no conflict of interest that would prejudice the impartiality of this scientific work.

\section{References}

Buaas FW, Krish AL, Sharma M, McLean DJ, Morris JL, Griswold MD, de Rooij DG \& Braun RE 2004 Plzf is required in adult male germ cells for stem cell self-renewal. Nature Genetics 36 $647-652$.

Chang L \& Karin M 2001 Mammalian MAP kinase signaling cascades. Nature $41037-40$.

Costoya JA, Hobbs RM, Barna M, Cattoretti G, Manova K, Sukhwani M, Orwig KE, Wolgemuth DJ \& Pandolfi PP 2004 Essential role of Plzf in maintenance of spermatogonial stem cells. Nature Genetics 36 653-659.

Eddy EM 2002 Male germ cell gene expression. Recent Progress in Hormone Research 57 103-128.

Elliott D 2003 Pathways of post-transcriptional gene regulation in mammalian germ cell development. Cytogenetics Genome Research 103 210-216.

Griswold MD 1998 The central role of Sertoli cells in spermatogenesis. Seminars in Cell and Developmental Biology 9 411-416.

Ha HY, Cho IH, Lee KW, Lee KW, Song JY, Kim KS, Yu YM, Lee JK, Song JS, Yang SD, Shin HS \& Han PL 2005 The axon guidance defect of the telencephalic commissures of the JSAP1-deficient brain was partially rescued by the transgenic expression of JIP1. Developmental Biology 277 184-199.

Hecht NB 1986 Regulation of gene expression during mammalian spermatogenesis. In Experimental Approaches to Mammalian Embryonic Development, pp 151-193. Eds J Rossant \& RA Pedersen. Cambridge: Cambridge University Press.

Ito $M$, Yoshioka $K$, Akechi $M$, Yamashita S, Takamatsu N, Sugiyama K, Hibi M, Nakabeppu Y, Shiba T \& Yamamoto KI 1999 JSAP1, a novel Jun $\mathrm{N}$-terminal protein kinase (JNK) that functions as scaffold factor in the JNK signaling pathway. Molecular and Cellular Biology $197539-7548$.

Ito $M$, Akechi M, Hirose R, Ichimura M, Takamatsu N, Xu P, Nakabeppu Y, Shiba T, Yamamoto KI \& Yoshioka K 2000 Isoforms of JSAP1 scaffold protein generated through alternative splicing. Gene 255 229-234.

Jagadish N, Rana R, Selvi R, Mishra D, Garg M, Yadav S, Herr JC, Okumura K, Hasegawa A, Koyama K \& Suri A 2005 Characterization of a novel human sperm-associated antigen 9 (SPAG9) having structural homology with c-Jun $\mathrm{N}$-terminal kinase-interacting protein. Biochemical Journal $38973-82$.

Kelkar N, Gupta S, Dickens M \& Davis RJ 2000 Interaction of a mitogen-activated protein kinase signaling module with the neuronal protein JIP3. Molecular and Cellular Biology 20 1030-1043.

Kelkar N, Demotte M-H, Weston CR, Barrett T, Sheppard BJ, Flavell RA \& Davis RJ 2003 Morphogenesis of the telencephalic commissure requires scaffold protein JNK-interacting protein 3 (JIP3). PNAS 100 9843-9848. 
Kelkar N, Standen CL \& Davis RJ 2005 Role of the JIP4 scaffold protein in the regulation of mitogen-activated protein kinase signaling pathways. Molecular and Cellular Biology 25 2733-2743.

Kyriakis JM \& Avruch J 2001 Mammalian mitogen-activated protein kinase signal transduction pathways activated by stress and inflammation. Physiological Reviews 81 807-769.

Lee CM, Onésime D, Reddy CD, Dhanasekaran N \& Reddy EP 2002 JLP: a scaffold protein that tethers JNK/p38MAPK signaling modules and transcription factors. PNAS 99 14189-14194.

Morrison DK \& Davis RJ 2003 Regulation of MAP kinase signaling modules by scaffold proteins in mammals. Annual Review of Cell and Developmental Biology 19 91-118.

Nagao Y 1989 Viability of meiotic prophase spermatocytes of rats is facilitated in primary culture of dispersed testicular cells on collagen gel by supplementing epinephrine or norepinephrine: evidence that meiotic prophase spermatocytes complete meiotic divisions. In Vitro Cellular and Developmental Biology 25 1088-1098.

Sato S, Ito M, Ito T \& Yoshioka K 2004 Scaffold protein JSAP1 is transported to growth cones of neuritis independent of JNK signaling pathways in PC12h cells. Gene 329 51-60.

Shima JE, McLean DJ, McCarrey JR \& Griswold MD 2004 The murine testicular transcriptome: characterizing gene expression in the testis during the progression of spermatogenesis. Biology of Reproduction 71 319-330.

Shiratsuchi A, Kawasaki Y, Ikemoto M, Arai Y \& Nakanishi Y 1999 Role of class B scavenger receptor type I in phagocytosis of apoptotic rat spermatogenic cells by Sertoli cells. Journal of Biological Chemistry 274 5901-5908.

Widmann C, Gibson S, Jarpe MB \& Johnson GL 1999 Mitogen-activated protein kinase: conservation of a three-kinase module from yeast to human. Physiological Reviews 79 143-180.

Xu P, Yoshioka K, Yoshimura D, Tominaga Y, Nishioka T, Ito M \& Nakabeppu Y 2003 In vitro development of mouse embryonic stem cells lacking JNK/stress-activated protein kinase-associated protein 1 (JSAP1) scaffold protein revealed its requirement during early embryonic neurogenesis. Journal of Biological Chemistry $\mathbf{2 7 8}$ $48422-48433$.

Yoshioka K 2004 Scaffold proteins in mammalian MAP kinase cascades. Journal of Biochemistry 135 657-661.

Received 26 August 2005

First decision 2 November 2005

Revised manuscript received 24 November 2005

Accepted 14 December 2005 